\title{
Sensor-Based Motion Planning for Autonomous Vehicle Teams
}

\author{
Sean Kragelund, Claire Walton, and Isaac Kaminer \\ Department of Mechanical \\ and Aerospace Engineering \\ Naval Postgraduate School \\ Monterey, CA 93943 \\ Email: spkragel@nps.edu, clwalton1@nps.edu,kaminer@nps.edu
}

\begin{abstract}
This paper employs a computational optimal control framework to develop a mission planning tool for a team of heterogeneous unmanned vehicles conducting a nominal mine countermeasures (MCM) mission. We first describe our motivation for developing vehicle-specific sensor models for unmanned surface and underwater vehicles working collaboratively to detect mines. Next, we describe the sonar detection models used to evaluate the performance of a long-range, forward-looking detection sonar and a high-resolution sidescan sonar deployed from these unmanned vehicles. Results from multiple computer simulations which highlight the flexibility and utility of this solution framework are presented.
\end{abstract}

\section{INTRODUCTION}

Over the last two decades, unmanned vehicle systems have grown steadily more capable, reliable, and ubiquitous, but most systems are still designed to conduct specific mission sets in a particular domain, with capabilities largely dependent on sensor payloads. But as system designers increasingly turn to commercial technologies and open architectures, it is easier than ever for robotic systems to inter-operate. As a result, multiple dissimilar vehicles can be combined into a collaborative team to overcome individual vehicle limitations and deliver advanced capabilities-even across operating domains. Autonomous vehicle teams have great potential in a wide range of scientific, commercial and defense applications, and they are especially well-suited for remote sensing in maritime domains.

To maximize the utility of a heterogeneous vehicle team for a given sensing mission, motion planning algorithms must consider the capabilities and limitations of each team member. At a minimum, they should incorporate dynamic and operational constraints to produce feasible trajectories. Optimization techniques can be used to allocate effort according to individual vehicles sensor performance. Such techniques can produce motion plans which are superior to conventional lawn mower survey patterns, which may be sub-optimal for certain sensors and infeasible for under-actuated vehicles to follow exactly.

Autonomous systems must also operate with imperfect information about their environment. This is particularly true in the maritime domain, where sensor accuracy usually depends on acoustic conditions and vehicle motion is subject to unknown disturbances at the water surface. In underwater search applications, the ability to detect and localize a target with sonar is impacted by several factors including acoustic noise, ambiguous geometry, and aspect-dependence. Consequently, the performance of an autonomous system may depend greatly on its ability to cope with uncertainty. Motion planning algorithms which consider uncertainty, therefore, can increase a system's overall robustness.

Optimal control provides a useful mathematical framework for solving motion planning problems with dynamic constraints and different performance criteria. Recent developments in numerical methods have made it possible to explicitly incorporate parameter uncertainty into the objective function of an an optimal control problem [1], [2], [3], [4]. Moreover, these generalized optimal control problems can incorporate sensor performance models to produce optimal vehicle trajectories for a given sensor configuration. Researchers have successfully applied these methods to solve motion planning problems with complex, multi-agent interactions in a variety of scenarios including optimal search, path coverage, and force protection [5].

In this paper, we employ this computational optimal control framework as a mission planning tool for a team of dissimilar vehicles conducting a nominal mine countermeasures (MCM) mission. We first describe our motivation behind developing vehicle-specific sensor models for Unmanned Surface Vessels (USVs) and Autonomous Underwater Vehicles (AUVs) working collaboratively to detect mines. Next, we describe the sonar detection models used to simulate the performance of a long-range, forward-looking sonar (FLS) and a high-resolution sidescan sonar (SS) deployed from these unmanned vehicles. Results from computer simulations which highlight the flexibility and utility of this solution framework are presented. Finally, we suggest directions for future research.

\section{Sonar Detection Models}

There are a number of complex sensing missions which could utilize autonomous vehicle teams to deliver a mix of different capabilities, particularly in environments which pose a risk to humans. The U.S. Navy has embraced this vision, and invested heavily in vehicle and sensor technologies for mine countermeasures (MCM). In general, MCM operations are 


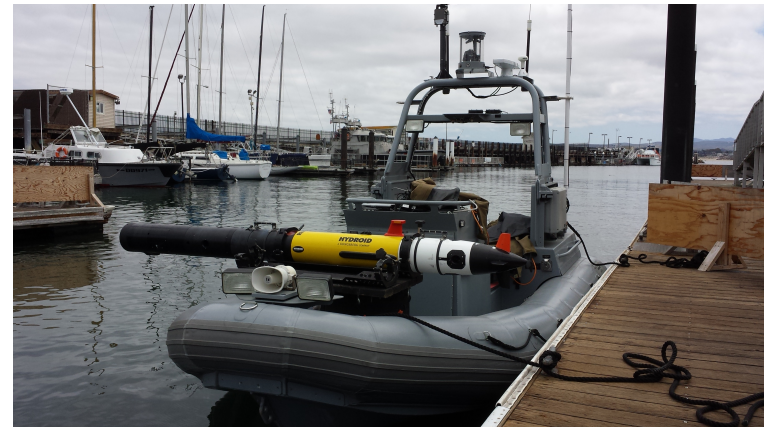

Fig. 1. The Naval Postgraduate School's SeaFox USV and REMUS 100 AUV

conducted in a sequence of phases, each performed by various types of vehicles and sensors [6]. Presently, these assets require dedicated support from manned platforms, but a current thrust of naval research is aimed at enabling autonomous systems to support other unmanned vehicles during MCM operations [7]. Fig. 1 shows a SeaFox USV and a REMUS 100 AUV, two of the fleet-representative vehicles used at the Naval Postgraduate School (NPS) Center for Autonomous Vehicle Research (CAVR). In this paper, we consider sensorbased motion planning strategies for a heterogeneous team comprised of these types of vehicles.

To address this problem of interest, we first develop models for two types of sensors routinely deployed in different phases of an MCM operation: 1) a long-range FLS for the target detection phase, and 2) a high-resolution SS for the subsequent reacquisition and identification phase. As these sensors are rigidly-mounted onto autonomous vehicles, models for detection performance should depend on individual vehicle dynamics and three-dimensional problem geometry, particularly when searching for bottom mines with FLS from a surface craft. Models should also reflect the region of poor sidescan coverage in the near-nadir area directly below an AUV's path. Many sensor models that have been widely-used in search theory do not address these geometric effects. Examples include "cookie cutter" models, which assume positive detection of all targets within a fixed range of the sensor, and lateral range curves, which graph detection probability versus the sensor's "point of closest approach" to the target [8], [9], [10].

The signal excess model, first proposed in [11] and still widely used for sonar performance modeling [12], [13], [14], simulates the conditions under which an active sonar system can detect an underwater mine-like object (MLO). This model assumes that detections will only occur when the acoustic energy transmitted by the sonar (ping) can overcome the twoway propagation losses in the environment such that the received signal (echo) exceeds a detection threshold. This signal excess can be computed using well-known sonar equations. The signal excess for an active sonar operating against a noise background is given in [10]:

$$
S E=S L-2 P L+T S-\left(N-A_{G}\right)-D T,
$$

where $S E=$ signal excess,

$P L=$ one-way propagation loss,

$T S=$ target strength,

$N=$ omni-directional sonar self-noise,

$A_{G}=$ array gain,

$D T=$ detection threshold.

An equivalent expression that includes signal processing terms for either frequency modulated (FM) or continuous wave (CW) pulse types can be found in [15]:

$$
\begin{aligned}
S E & =S L-2 P L+T S-(N-D I+10 \log B) \\
& -(5 \log d-10 \log B T-5 \log n),
\end{aligned}
$$

where $D I=$ directivity index,

$B=$ pulse bandwidth $[\mathrm{Hz}]$,

$T=$ pulse duration $[\mathrm{s}]$,

$d=$ detection index,

$n=$ number of pings used in detection decisions.

Each term in (1) and (2) are expressed in $\mathrm{dB}$, unless otherwise specified. For sonar performance analysis, the terms of the signal excess equation can be combined into two terms:

$$
\begin{aligned}
S E(t) & =F O M-P L(D(t)) \\
\text { where } \mathrm{FOM} & =\text { Figure of Merit, } \\
P L & =\text { Propagation Losses, and } \\
D & =\text { Distance from the target. }
\end{aligned}
$$

Figure of Merit is useful for analyzing the performance of different passive or noise-limited active sonar designs, as this metric is independent of range or a specific operating region for these cases (unlike reverberation-limited active sonar). Even though exact design parameters for Navy sonars are difficult to obtain (and potentially classified), a Figure of Merit suitable for relative performance analysis can still be estimated from sonar design reference manuals [15] or commercial sonar specifications [16], [17]. The signal excess of a given sonar's FOM is especially attractive for simulating detections along a moving vehicle's trajectory $\vec{x}(t)$, since it depends only on the distance between the vehicle and the mine location $\vec{\omega}$ at each moment in time: $D(t)=\left\|\left[\begin{array}{lll}d x & d y & d z\end{array}\right]^{T}\right\|=\|\vec{\omega}-\vec{x}(t)\|$. Here the uncertain mine location $\vec{\omega}$ is characterized by the probability density function $\phi: \Omega \rightarrow \mathbb{R}$. We assume that propagation losses are due primarily to spherical spreading and absorption of the acoustic energy as it travels through the water, "a useful working rule for initial design and performance comparisons" [15]. In the following equation, $a$ is the frequency-dependent absorption coefficient of seawater.

$$
P L(\vec{x}(t), \vec{\omega})=20 \log _{10}(\|\vec{\omega}-\vec{x}(t)\|)+a\|\vec{\omega}-\vec{x}(t)\|
$$

Tabulated values of $a$ for different frequencies can be found in sonar design references such as [15] and [18], while [19] 


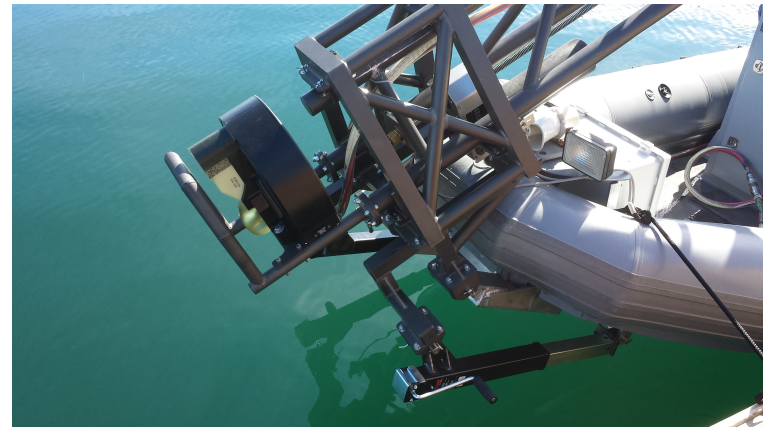

Fig. 2. An ATLAS sonar mounted on the NPS SeaFox USV

provides an equation for $a$ in $\mathrm{dB} / \mathrm{km}$ as a function of frequency in $\mathrm{kHz}$ :

$$
a=\frac{0.11 f^{2}}{1+f^{2}}+\frac{44 f^{2}}{4100+f^{2}}+0.0003 f^{2}+0.003
$$

If we assume that signal excess is a normally-distributed random variable with variance $\sigma^{2}$, the instantaneous probability of detection for a single glimpse with the sonar can be written in terms of its cumulative normal distribution $\Phi$ :

$$
p(\vec{x}(t), \vec{\omega})=\Phi\left(\frac{S E(\vec{x}(t), \vec{\omega})}{\sigma}\right)
$$

with typical values of $\sigma$ lying between $3 \mathrm{~dB}$ and $9 \mathrm{~dB}$ [8], [10], [20]. Practically speaking, at times when $P L=F O M$, detection probability is $p(t)=0.5$, i.e., the sonar has an equal probability of either detecting or missing a mine. We further assume that detection can be modeled by a Poisson process in which detection opportunities, or glimpses, occur at rate $\lambda$, producing the detection rate $D R(\vec{x}(t), \vec{\omega})=\lambda p(\vec{x}(t), \vec{\omega})$ [8].

\section{A. Forward Looking Sonar}

A Figure of Merit for a long range, forward-looking sonar (FLS) similar to the Autonomous Topographic Large Area Sonar (ATLAS) mounted on the NPS SeaFox USV (Fig. 2) can be computed from a nominal design specification. This type of sonar is used to perform wide-area mine detection during the first phase of mine countermeasures (MCM) operations [6]. Following the design example for a mine-hunting sonar in [15], we can, for example, specify a $200 \mathrm{kHz}$ sonar with 120-degree horizontal field of view, 5-degree vertical field of view, and nominal operating range of 400 meters [21]. From the specified operating frequency and desired beam widths we can compute the directivity indices for a linear transmit array and cylindrical receive array. Assuming the sonar operates at $10 \mathrm{~W}$ power, we compute a transmitter source level of $197 \mathrm{~dB}$. The source level, array geometry, and FM signal processing in this design yield a FOM of $72 \mathrm{~dB}$ as shown in Table I, where all specified or assumed parameters are italicized.

\section{B. Sidescan Sonar}

Next, we estimate a Figure of Merit for a short range, sidelooking sonar similar to the sidescan sonar used on the NPS REMUS 100 AUV. This type of sensor is representative of
TABLE I

\begin{tabular}{|c|c|c|}
\hline & FLS & Sidescan \\
\hline Specified or Assumed & & \\
\hline NominalRange & $400 \mathrm{~m}$ & $40 \mathrm{~m}$ \\
\hline Frequency & $200 \mathrm{kHz}$ & $900 \mathrm{kHz}$ \\
\hline $\begin{array}{c}\text { Transmit Beam Widths } \\
\quad \text { Horizontal } \\
\text { Vertical }\end{array}$ & $\begin{array}{c}120^{\circ} \\
5^{\circ}\end{array}$ & $\begin{array}{l}0.4^{\circ} \\
40^{\circ}\end{array}$ \\
\hline $\begin{array}{l}\text { Receive Beam Widths } \\
\quad \text { Horizontal } \\
\text { Vertical } \\
\end{array}$ & $\begin{array}{l}2^{\circ} \\
2^{\circ}\end{array}$ & $\begin{array}{l}0.4^{\circ} \\
40^{\circ}\end{array}$ \\
\hline Propagation Loss, $P L$ & $71 \mathrm{~dB}$ at $400 \mathrm{~m}$ & $43 d B$ at $40 m$ \\
\hline $\begin{array}{l}\text { Pulse Length, T } \\
\qquad F M \\
\text { CW }\end{array}$ & $\begin{array}{c}10 \mathrm{~ms} \\
-\end{array}$ & $\begin{array}{c}- \\
6.67 \mu s\end{array}$ \\
\hline $\begin{array}{l}\text { Pulse Bandwidth, B } \\
\quad F M \\
\text { CW }\end{array}$ & $\begin{array}{c}80 \mathrm{kHz} \\
-\end{array}$ & $\frac{-}{150 \mathrm{kHz}}$ \\
\hline $5 \log d$ & $10 d B$ & $3 d B$ \\
\hline $5 \log n$ & $10 d B$ & $3 d B$ \\
\hline Computed & & \\
\hline $\begin{array}{l}\text { Directivity Index, DI } \\
\text { Transmit } \\
\text { Receive }\end{array}$ & $\begin{array}{l}16 \mathrm{~dB} \\
35 \mathrm{~dB}\end{array}$ & $\begin{array}{l}27 \mathrm{~dB} \\
27 \mathrm{~dB}\end{array}$ \\
\hline Source Level, SL & $197 \mathrm{~dB}$ & $204 \mathrm{~dB}$ \\
\hline Ambient or Self-Noise, $\mathrm{N}$ & $35 \mathrm{~dB}$ & $44 \mathrm{~dB}$ \\
\hline Target Strength, TS & $-30 \mathrm{~dB}$ & $-30 \mathrm{~dB}$ \\
\hline Figure of Merit, FOM & $72 \mathrm{~dB}$ & $49 \mathrm{~dB}$ \\
\hline
\end{tabular}

ACTIVE SONAR DESIGN PARAMETERS FOR NOISE-LIMITED FOM

high-resolution sonar used to reacquire previously-detected MLOs and identify them for subsequent neutralization [6]. Following the design example for a sidescan sonar in [15], and using manufacturer specifications for a $900 \mathrm{kHz}$ sidescan sonar [16], we can compute a FOM for this sensor as well. Assuming the sonar operates at $4 \mathrm{~W}$, we compute a transmitter source level of $204 \mathrm{~dB}$. The source level, array geometry, and $\mathrm{CW}$ signal processing in this design yield a FOM of $49 \mathrm{~dB}$ as shown in Table I, where all specified or assumed parameters are italicized.

\section{Geometry Impacts}

The signal excess detection model developed thus far, for a given sonar's Figure of Merit, depends only on the omnidirectional distance to the MLO of interest. This is equivalent to other popular sonar detection models used in search theory such as the definite range model or lateral range curves. Although useful for computing a constant sweep width for coverage planning algorithms [9], both of these models fail to address regions of negligible detection performance located directly below an underwater vehicle using sidescan sonar or a surface craft using forward-looking sonar. Furthermore, most physical sonar systems are designed with a specific beam pattern and do not perform equally well in all directions. 
Therefore, to more accurately estimate a sonar's true detection performance when mounted on a vehicle, we enforce its beam geometry in three dimensions. This is achieved by defining horizontal and vertical fields of view (FOV) relative to the vehicle's body-fixed axes, and degrading detection performance outside of these limits. First, we define the horizontal FOV to lie between its lower and upper azimuth angles in the horizontal plane, denoted $\alpha_{L}$ and $\alpha_{U}$, respectively, where the superscript $b$ indicates angles resolved in the body-fixed reference frame.

$$
{ }^{b} \alpha_{L}=-\frac{\alpha_{F O V}}{2}, \quad{ }^{b} \alpha_{U}=+\frac{\alpha_{F O V}}{2}
$$

Likewise the vertical FOV is defined between its lower and upper elevation angles in the vertical plane, denoted $\varepsilon_{L}$ and $\varepsilon_{U}$, respectively.

$$
{ }^{b} \varepsilon_{L}=\varepsilon_{D E}-\frac{\varepsilon_{F O V}}{2}, \quad{ }^{b} \varepsilon_{U}=\varepsilon_{D E}+\frac{\varepsilon_{F O V}}{2}
$$

where $\varepsilon_{D E}$ is a fixed downward elevation angle selected to ensure that the sonar can ensonify the sea floor. The azimuth angle and elevation angle between the vehicle and MLO of interest in the inertial navigation frame, denoted by the superscript $n$, are computed as:

$$
\begin{aligned}
{ }^{n} \alpha= & \operatorname{atan} 2\left(\omega_{y}-y, \omega_{x}-x\right)=\operatorname{atan} 2(d y, d x) \\
{ }^{n} \varepsilon & =\arctan \left(\frac{-\omega_{z}-0}{\sqrt{\left(\omega_{x}-x\right)^{2}+\left(\omega_{y}-y\right)^{2}}}\right) \\
& =\arctan \left(\frac{-d z}{\sqrt{d x^{2}+d y^{2}}}\right)
\end{aligned}
$$

To determine the azimuth angle to a potential mine in the sonar's FOV, the vector from the vehicle to the MLO must be rotated from the navigation frame into the vehicle's body-fixed reference frame. For a vehicle at heading angle $\psi$, the rotated vector is:

$$
\begin{gathered}
{ }^{b} d x={ }^{n} d x \cos (\psi)+{ }^{n} d y \sin (\psi) \\
{ }^{b} d y=-{ }^{n} d x \sin (\psi)+{ }^{n} d y \cos (\psi) \\
{ }^{b} \alpha=\operatorname{atan} 2\left({ }^{b} d y,{ }^{b} d x\right)
\end{gathered}
$$

Using these angles, we define scalar masking functions that degrade detection performance for mines with azimuth (elevation) angles that lie outside the sonar's horizontal (vertical) field of view. Each masking factor is constructed from two sigmoidal functions: one that smoothly transitions the scalar multiplier value from 0 to 1 as a mine "enters" the lower FOV boundary, and one that transitions it back down to 0 as a mine "exits" the upper FOV boundary. The azimuth and elevation masking functions are given in (14) and (15), respectively.

$$
F_{\alpha}(\vec{x}(t), \vec{\omega})=\frac{1}{1+e^{p_{\alpha}\left(\alpha_{L}-{ }^{b} \alpha\right)}}+\frac{1}{1+e^{p_{\alpha}\left({ }^{b} \alpha-\alpha_{U}\right)}}-1
$$

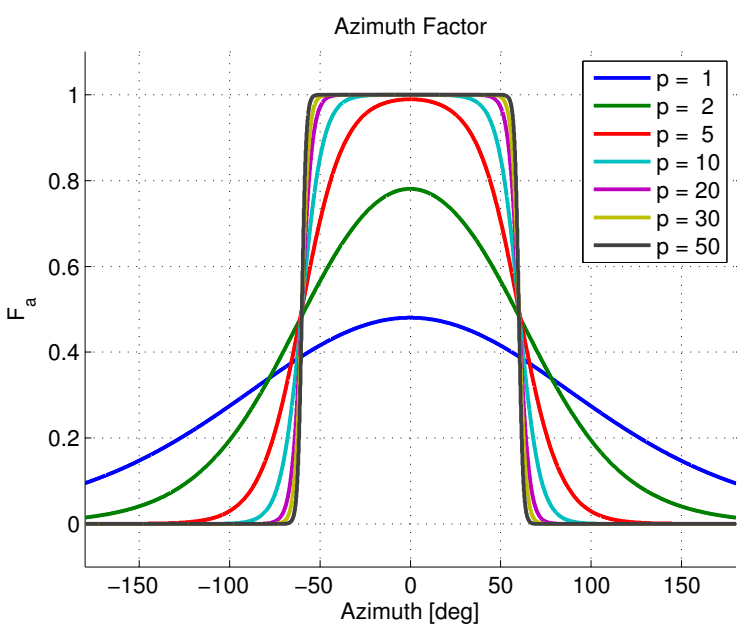

Fig. 3. $F_{\alpha}$ vs. azimuth angle and $p_{\alpha}$ for a 120-degree horizontal FOV

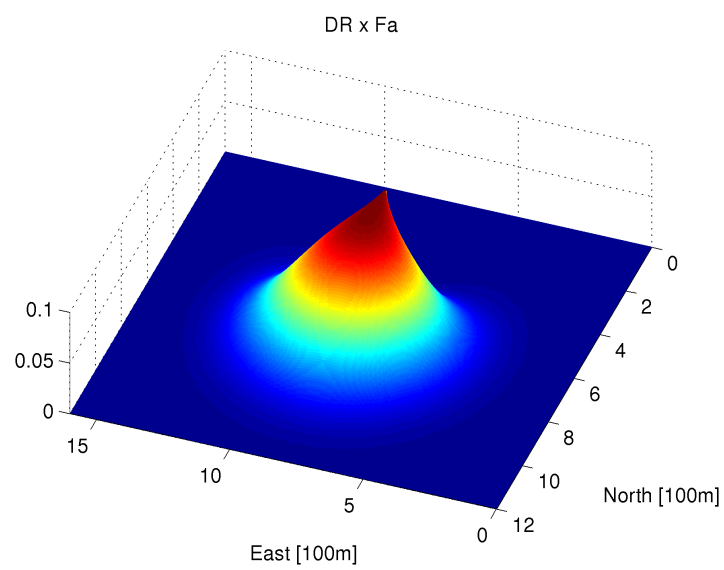

Fig. 4. $D R$ vs. azimuth angle only for a 120-degree horizontal FOV

$$
F_{\varepsilon}(\vec{x}(t), \vec{\omega})=\frac{1}{1+e^{p_{\varepsilon}\left(\varepsilon_{L}-\varepsilon\right)}}+\frac{1}{1+e^{p_{\varepsilon}\left(\varepsilon-\alpha_{U}\right)}}-1
$$

The $p_{\alpha}$ and $p_{\varepsilon}$ parameters can be tuned to adjust the growth rate of their respective sigmoid functions. Applying these masking functions results in the geometry-dependent sonar detection rate $D R(\vec{x}(t), \vec{\omega})=\lambda p(\vec{x}(t), \vec{\omega}) F_{\alpha}(\vec{x}(t), \vec{\omega}) F_{\varepsilon}(\vec{x}(t), \vec{\omega})$. Fig. 4 illustrates this noise-limited detection rate as a function of target position relative to a forward-looking sonar located at $(400 \mathrm{~m}, 800 \mathrm{~m})$ with azimuth masking factor $F_{\alpha}(\vec{x}(t), \vec{\omega})$ corresponding to a 120-degree horizontal field of view. The color scale ranges from a detection probability of 0 (blue) to 1 (dark red). The effect of the elevation masking factor is ignored for this plot (e.g., $F_{\varepsilon}(\vec{x}(t), \vec{\omega})=1$ ).

Because many sonar systems, e.g. sidescan sonar, perform poorly during turning maneuvers, another scalar term is applied to degrade detection performance as a function of vehicle turn rate, $r(t)$. A Gaussian-like function which reaches a maximum value of 1 for straight line motion is

$$
F_{r}(\vec{x}(t))=e^{-\frac{1}{2}\left[\frac{r(t)}{\sigma_{r}}\right]^{2}} .
$$


This modifier is used to account for the adverse effects of non-zero turn rate. It can be adjusted by the tuning parameter $\sigma_{r}$ and produces the sidescan sonar detection rate:

$$
D R(\vec{x}(t), \vec{\omega})=\lambda p(\vec{x}(t), \vec{\omega}) F_{\alpha}(\vec{x}(t), \vec{\omega}) F_{\varepsilon}(\vec{x}(t), \vec{\omega}) F_{r}(\vec{x}(t)) .
$$

A primary consideration in selecting these scalar functions is their numerical smoothness, which greatly aids numeric gradient computations, and their tuning parameters can be adjusted to reflect most sonar geometries.

\section{Optimal Search Objective Function}

In this section we derive the probability that a vehicle's sonar completely fails to detect a mine using the sonar detection rate function obtained above. Minimizing this objective function will thereby maximize the probability that the sonar successfully detects the mine. In this framework, we assume that the detection probability on a sufficiently small time interval $[t, t+\Delta t]$ is $P_{D}(\Delta t)=D R(\vec{x}(t), \vec{\omega}) \Delta t$ [8]. We also assume that this quantity is independent of the detection probability on all prior time intervals. Therefore, using the complement, the probability of not detecting a mine at time $t+\Delta t$ is

$$
\begin{aligned}
P_{N D}(t+\Delta t) & =P_{N D}(t)\left[1-P_{D}(\Delta t)\right] \\
& =P_{N D}(t)[1-D R(\vec{x}(t), \vec{\omega}) \Delta t] .
\end{aligned}
$$

This equation can be rearranged as the difference equation

$$
\frac{P_{N D}(t+\Delta t)-P_{N D}(t)}{\Delta t}=-P_{N D}(t) D R(\vec{x}(t), \vec{\omega}) .
$$

In the limit as $\Delta t \rightarrow 0$ we obtain

$$
\dot{P}_{N D}(t)=-P_{N D}(t) D R(\vec{x}(t), \vec{\omega}),
$$

which has the closed form solution:

$$
P_{N D}(t)=e^{-\int_{0}^{T_{f}} D R(\vec{x}(\tau), \vec{\omega}) d \tau}
$$

In this paper, we seek to minimize the expected probability that a sonar fails to detect a mine at the uncertain mine location $\vec{\omega}$ characterized by the probability density function $\phi$ defined in Section II [5]. Therefore, the cost function becomes

$$
J=\mathbb{E}\left\{P_{N D}\left(T_{f}\right)\right\}=\int_{\Omega} e^{-\int_{0}^{T_{f}} D R(\vec{x}(\tau), \vec{\omega}) d \tau} \phi(\vec{\omega}) d \vec{\omega} .
$$

Note, the cost $J$ is a function of the uncertain parameter $\vec{\omega}$ and has the standard form of the running cost in the generalized optimal control problem formulation described in [1] .

\section{Optimal MCM SEARCh Benchmark Problem}

\section{A. Vehicle Dynamics}

We simulate an Unmanned Surface Vehicle (USV) with bow-mounted FLS conducting an initial MCM search at constant velocity using the following dynamic equations of motion (EOM):

$$
\begin{aligned}
\dot{x}(t) & =V \cos (\psi(t)) \\
\dot{y}(t) & =V \sin (\psi(t)) \\
\dot{\psi}(t) & =r(t) \\
\dot{r}(t) & =\frac{1}{T}(K u(t)-r(t)) \\
\text { where } \mathrm{u}(\mathrm{t}) & =\delta_{\text {rudder }}(t)
\end{aligned}
$$

These equations implement a simple, slow-speed Nomoto turning model in state space form: $\dot{\vec{x}}(t)=f(\vec{x}(t), \vec{u}(t))$ with state vector $\vec{x} \equiv[x, y, \psi, r]^{T}$. The parameters $K[1 / \mathrm{s}]$ and $T$ [s] can be identified as described in [22]. The state variables $(x, y)$ define the vehicle's position in meters along the (north, east) axes of the navigation reference frame; $\psi$ describes the vehicle's heading angle in radians measured clockwise from the North axis; and $r$ is the vehicle's turn rate in radians per second. The vehicle travels with constant forward velocity $V$ measured along the body-fixed x-axis, of $2.5 \mathrm{~m} / \mathrm{s}$. For simplicity, we use these same equations of motion (23), with constant velocity $V=1.5 \mathrm{~m} / \mathrm{s}$ and constant depth $z=3 \mathrm{~m}$, to simulate AUV motion. More accurate AUV dynamic equations are presented in [23].

\section{B. Problem Scaling}

We wish to solve this optimal search problem numerically, but the domains of our state variables and uncertain parameters have different orders of magnitude. It is important to properly scale the problem before applying a numeric solver. This can be achieved by defining canonical units for distance, time, etc. and transforming the original problem variables into nondimensional versions with similar domains [24]. For this problem, we define the canonical distance unit $D U=100$ meters, and the canonical time unit $T U=100$ seconds. This produces the canonical velocity unit of $V U=\frac{D U}{T U}=1 \mathrm{~m} / \mathrm{s}$. We can restate our state variables in canonical units as follows:

$$
\bar{x}=\frac{x}{D U}, \quad \bar{y}=\frac{y}{D U}, \bar{\psi}=\psi, \bar{r}=\frac{r}{1 / T U}, \bar{t}=\frac{t}{T U}
$$

Our chosen scaling must also be applied to the Nomoto parameters and control input:

$$
\bar{K}=(T U) K, \bar{T}=\frac{T}{T U}, \bar{u}=u
$$

Substituting these expressions into our original dynamics for $x$ and $y$ yields their state space equations in canonical units:

$$
\begin{aligned}
\dot{x}=\frac{d x}{d t}=\frac{d(D U \bar{x})}{d(T U \bar{t})}=\frac{D U}{T U} \frac{d \bar{x}}{d \bar{t}} & =V U \dot{\bar{x}} \\
\dot{\bar{x}}=\frac{1}{V U} \dot{x}=\frac{1}{V U} V \cos (\psi) & =\bar{V} \cos (\bar{\psi}) \\
\text { so, by similarity } \dot{\overline{\mathrm{y}}} & =\bar{V} \sin (\bar{\psi})
\end{aligned}
$$


Likewise, substituting canonical units for $\psi$ and $r$ yields:

$$
\begin{aligned}
\dot{\psi} & =\frac{d \psi}{d t}=\frac{d \bar{\psi}}{d(T U \bar{t})}=\frac{1}{T U} \frac{d \bar{\psi}}{d \bar{t}}=\frac{1}{T U} \dot{\bar{\psi}} \\
\dot{\bar{\psi}} & =T U \dot{\psi}=(T U) r=\bar{r} \\
\dot{r} & =\frac{d r}{d t}=\frac{d(1 / T U) \bar{r}}{d(T U \bar{t})}=\frac{1}{T U^{2}} \frac{d \bar{r}}{d \bar{t}}=\frac{1}{T U^{2}} \dot{\bar{r}} \\
\dot{\bar{r}} & =\left(T U^{2}\right) \dot{r}=\left(T U^{2}\right) \frac{1}{T}(K u-r) \\
& =\left(T U^{2}\right) \frac{1}{T U \bar{T}}\left(\frac{\bar{K}}{T U} \bar{u}-\frac{\bar{r}}{T U}\right) \\
& =\frac{1}{\bar{T}}(\bar{K} \bar{u}-\bar{r})
\end{aligned}
$$

Equations (26) through (29) confirm that our scaling has not changed the underlying dynamics of the problem. However, the expression for propagation loss (PL) in the detection rate equation must be modified slightly when using canonical units. Recall from (4) that propagation loss includes a spherical spreading term, $20 \log _{10}(\|\vec{\omega}-\vec{x}(t)\|)$, and an acoustic absorption term $a\|\vec{\omega}-\vec{x}(t)\|$. At each time $t$ we have:

$$
\begin{aligned}
& D=\|\vec{\omega}-\vec{x}\| \\
& D=\sqrt{\left(\omega_{x}-x\right)^{2}+\left(\omega_{y}-y\right)^{2}+\left(\omega_{z}-z\right)^{2}} \\
& D=\sqrt{(d x)^{2}+(d y)^{2}+(d z)^{2}} \\
& D=\sqrt{(D U \overline{d x})^{2}+(D U \overline{d y})^{2}+(D U \overline{d z})^{2}} \\
& D=D U \sqrt{\overline{d x}^{2}+\overline{d y}^{2}+\overline{d z}^{2}} \\
& D=D U \bar{D}
\end{aligned}
$$

Since $P L=20 \log _{10}(D)+a \cdot D$, we define $\overline{P L}$ using canonical units as:

$$
\begin{aligned}
& \overline{P L}=20 \log _{10}(D U \bar{D})+a(D U \bar{D}) \\
& \overline{P L}=20 \log _{10}(D U)+20 \log _{10}(\bar{D})+\bar{a}(\bar{D})
\end{aligned}
$$$$
\text { where } \bar{a}=D U a
$$

\section{Simulation Results}

\section{A. Sonar Detection Model Testing}

The sonar models developed in Section II were tested in simulation to verify their performance. Fig. 5 shows a USV with FLS as it moves along an open loop trajectory through a square search area. The color at each location in the search area designates $P_{N D}\left(T_{f}\right)$, the probability that the sonar failed to detect a mine at that location before the end of the mission. This is our measure of MCM risk. Assuming that we have no prior information and that a possible mine location is uniformly distributed within the search area, the maximum $P_{N D}\left(T_{f}\right)$ value (dark red) is a function of search area size. We note that in areas covered by the USV's sonar along this trajectory, $P_{N D}\left(T_{f}\right)$ approaches zero (blue), meaning there is a high probability that the sonar would have detected a mine in those areas.

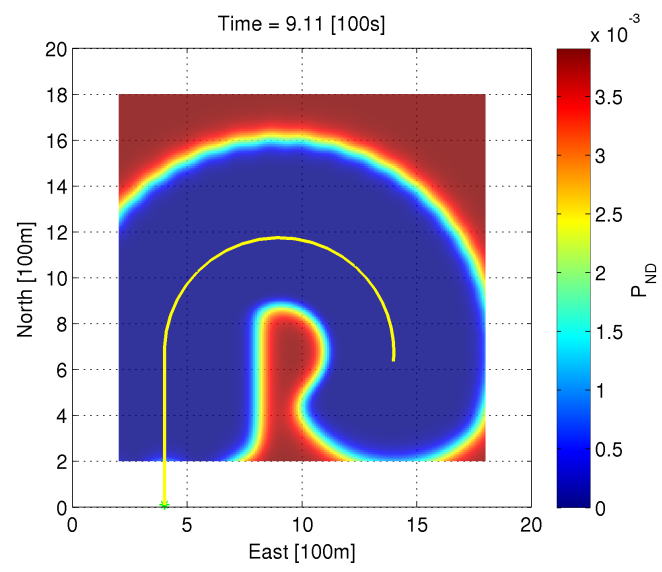

Fig. 5. Simulated search with USV and forward looking sonar

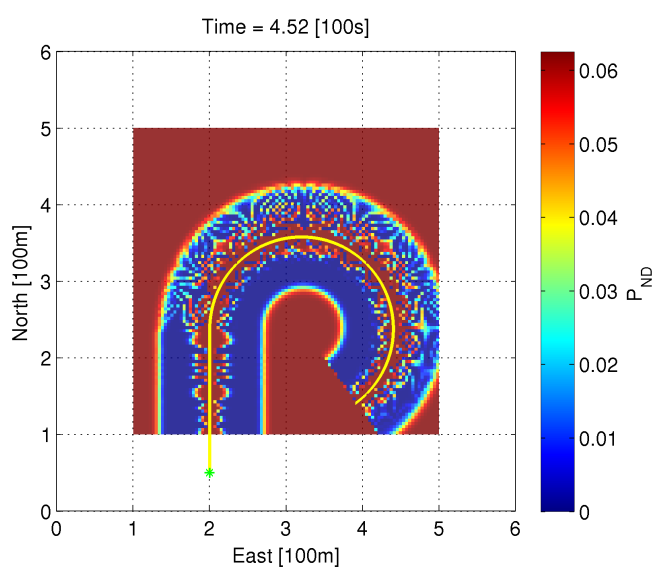

Fig. 6. Simulated search with AUV and sidescan sonar

A similar trajectory is shown for an AUV with sidescan sonar in Fig. 6. To accommodate the slower AUV and shorter range sonar, the designated search area is much smaller than the FLS search area in Fig. 5. While the corresponding maximum values for $P_{N D}\left(T_{f}\right)$ are higher in a given location, the relative color scale along the vehicle's trajectory indicates that our sidescan detection model is consistent with actual sidescan performance, e.g. it provides little to no coverage beneath the vehicle and degraded performance when the vehicle is turning.

\section{B. Time-Limited MCM Search}

The remainder of this paper will focus on optimal motion planning for mine detection by one or two USVs equipped with forward looking sonar (FLS). Establishing "benchmark" problems based on computational optimal search can facilitate comparisons between different vehicle types and sensor payloads to assess the best team composition for a given mission. For this problem, we wish to detect mines on a flat sea floor in 20 meters of water within a $1.6 \times 1.6 \mathrm{~km}^{2}$ search area. If the USV bow-mounted FLS has FOM $=43.5 \mathrm{~dB}$, we wish to compute the optimal trajectories that minimize $P_{N D}\left(T_{f}\right)$ for fixed $T_{f}=1500$ seconds. 


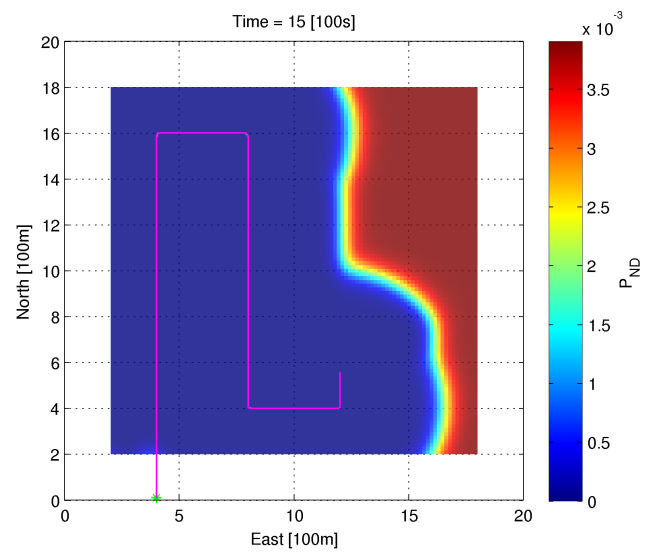

Fig. 7. Time-limited search by one USV using a manual lawnmower pattern

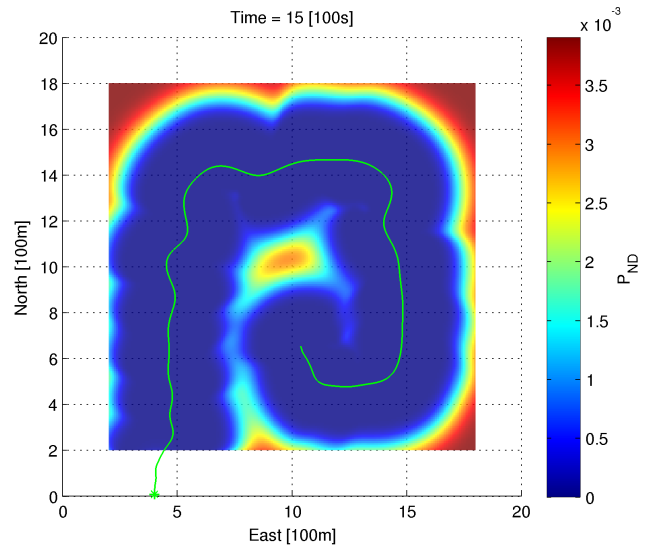

Fig. 8. Optimal time-limited search by one USV

The fixed $T_{f}$ constraint makes it extremely difficult to manually program a lawnmower survey pattern with complete coverage using only one USV. The pattern shown in Fig. 7 still leaves $P_{N D}\left(T_{f}\right)=0.34$. Potential solutions include deploying a better sonar (with a higher FOM) and increasing the lawnmower pattern's row spacing, or adding additional team members. Both solutions would likely increase the overall system cost. Alternatively, we find that the optimal solution to our single-USV benchmark problem (Fig. 8) achieves $P_{N D}\left(T_{f}\right)=0.16$. This level of risk may be acceptable in certain situations; if not, we can easily compute a motion plan for two USVs (Fig. 9) that achieves $P_{N D}\left(T_{f}\right)=0.03$ in $T_{f}=1000$ seconds, or $33 \%$ less time than the original time allotted.

\section{CONCLUSiON}

The key contribution of this paper is a physics-based sonar detection rate model for mine countermeasures (MCM) that can be used to compute the expected probability that search vehicles fail to detect all mines in an area of interest. This cost function represents our measure of risk associated with MCM and is minimized over all vehicle trajectories that

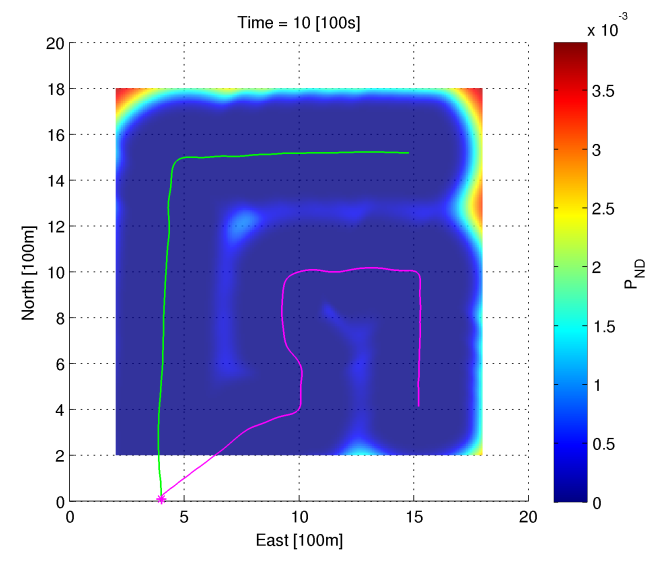

Fig. 9. Optimal time-limited search by two USV

satisfy constraints imposed by the dynamic limitations of the vehicles. A number of simple examples were presented which suggest that our computational framework is suitable for optimal motion planning applications with spatial and dynamic constraints, as well as parameter uncertainty. The authors are actively developing a mission planning tool for MCM based on this computational framework, as well as working to expand the class of problems that can solved using this approach.

\section{ACKNOWLEDGMENT}

The authors would like to thank the Consortium for Robotics and Unmanned Systems Education and Research (CRUSER) at the Naval Postgraduate School for supporting this research.

\section{REFERENCES}

[1] C. Phelps, Q. Gong, J. O. Royset, C. Walton, and I. Kaminer, "Consistent approximation of a nonlinear optimal control problem with uncertain parameters," Automatica, vol. 50, no. 12, pp. 2987-2997, Dec. 2014.

[2] J. Foraker, J. O. Royset, and I. Kaminer, "Search-Trajectory Optimization: Part I, Formulation and Theory," Journal of Optimization Theory and Applications, Jun. 2015.

[3] — , "Search-Trajectory Optimization: Part II, Algorithms and Computations," Journal of Optimization Theory and Applications, Jun. 2015.

[4] C. Walton, C. Phelps, Q. Gong, and I. Kaminer, "A numerical algorithm for optimal control of systems with parameter uncertainty," in Proceedings of the 10th IFAC Symposium on Nonlinear Control (NOLCOS), Monterey, CA, 2016.

[5] C. L. Walton, Q. Gong, I. Kaminer, and J. O. Royset, "Optimal motion planning for searching for uncertain targets," IFAC Proceedings Volumes, vol. 47, no. 3, pp. 8977 - 8982, 2014, 19th IFAC World Congress.

[6] D. Sternlicht, J. Fernandez, R. Holtzapple, D. Kucik, T. Montgomery, and C. Loeffler, "Advanced sonar technologies for autonomous mine countermeasures," in OCEANS 2011, Sep. 2011, pp. 1-5.

[7] J. Keller, "Raytheon, Applied Physical Sciences to develop Navy unmanned mine-hunting technology." [Online]. Available: http://www. militaryaerospace.com/articles/2013/06/mine-hunting-USV.html

[8] A. R. Washburn, Search and Detection, 4th ed. Linthicum, MD: Institute for Operations Research and the Management Sciences, 2002.

[9] A. Washburn and M. Kress, Combat Modeling. Boston, MA: Springer, 2009.

[10] D. H. Wagner, W. C. Mylander, T. J. Sanders, and United States Naval Academy, Eds., Naval operations analysis, 3rd ed. Annapolis, Md: Naval Institute Press, 1999.

[11] B. Koopman, Search and Screening. Naval Historical Center, 1946.

[12] P. Etter, Underwater Acoustic Modeling and Simulation, 4th ed. CRC Press, 2013. 
[13] R. E. Keenan, "An introduction to GRAB eigenrays and CASS reverberation and signal excess," in OCEANS 2000 MTS/IEEE Conference and Exhibition, vol. 2, 2000, pp. 1065-1070 vol.2.

[14] P. C. Chu, C. Cintron, S. D. Haeger, and R. E. Keenan, "Acoustic Mine Detection Using the Navy's CASS/GRAB Model," in Fifth International Symposium on Technology and the Mine Problem. Monterey, CA: Society for Counter-Ordnance Technology/Mine Warfare Association, 2002.

[15] A. D. Waite, Sonar for Practising Engineers. Wiley, May 2002

[16] "Sea Scan SSPC AUV System | Marine Sonic Technology." [Online]. Available: http://www.marinesonic.com/products/ seascanSSPCAUV.html

[17] "EdgeTech Customer \& Technical Support | Resource Center." [Online]. Available: http://www.edgetech.com/underwater-technology-support/ \#resource-center

[18] M. Ainslie, Principles of Sonar Performance Modelling. Berlin: Springer, 2010.
[19] R. J. Urick, Principles of Underwater Sound 3rd Edition, 3rd ed. Peninsula Pub, Aug. 1996.

[20] P. C. Chu, N. A. Vares, and R. E. Keenan, "Uncertainty in acoustic mine detection due to environmental variability," in Sixth Monterey International Symposium on Technology and the Mine Problem. Monterey, CA: Mine Warfare Association, 2004.

[21] C. Loeffler, "Thunder Bay 2010: Cutting Edge Technology and the Hunt for Lake Hurons Lost Ships." [Online]. Available: http://oceanexplorer. noaa.gov/explorations/10thunderbay/background/sonar/sonar.html

[22] C. Sonnenburg, A. Gadre, D. Horner, S. Kragelund, A. Marcus, D. J. Stilwell, and C. A. Woolsey, "Control-oriented planar motion modeling of unmanned surface vehicles," Virginia Center for Autonomous Systems, Tech. Rep. VACAS-2010-01, 2010. [Online]. Available: http://www.unmanned.vt.edu/discovery/reports/VaCAS_2010_01.pdf

[23] D. Sgarioto, "Control system Design and Development for the REMUS Autonomous Underwater Vehicle," Defence Technology Agency, Tech. Rep. DTA Report 240, May 2007.

[24] I. M. Ross, "AE4850 Astrodynamic Optimization Course Notes," Naval Postgraduate School, Monterey, CA, 2010. 